\title{
Medidas para a promoção da bicicleta em São Paulo: uma análise Multicritério e Multiatores
}

\author{
Measures for cycling promotion in São Paulo: a Multi Actor Multi Criteria \\ Analysis
}

\author{
Hellem de Freitas Miranda ${ }^{1}$, Antônio Nélson Rodrigues da Silva ${ }^{2}$, Karst T. Geurs ${ }^{3}$, \\ Anna Beatriz Grigolon ${ }^{4}$ \\ 1Universidade de São Paulo, São Paulo - Brasil, miranda.hf@gmail.com \\ 2Universidade de São Paulo, São Paulo - Brasil, anelson@sc.usp.br \\ 3University of Twente, Enschede - Países Baixos, k.t.geurs@utwente.nl \\ ${ }^{4}$ University of Twente, Enschede - Países Baixos, a.b.grigolon@utwente.nl
}

\section{Recebido:}

29 de fevereiro de 2020

Aceito para publicação:

4 de novembro de 2020

Publicado:

30 de abril de 2021

Editor de área:

Helena Beatriz Cybis

\section{Palavras-chave:}

Bicicleta.

MAMCA.

Análise multicritério e multiatores.

São Paulo.

Keywords:

Bicycle.

MAMCA.

Multi actor multi criteria analysis.

São Paulo.

DOI:10.14295/transportes.v29i1.2316

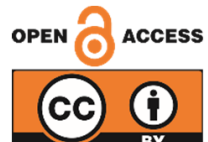

\begin{abstract}
RESUMO
Esse estudo, cujo objetivo é identificar barreiras e incentivos para o uso da bicicleta na cidade de São Paulo, a partir da consulta a stakeholders e da adaptação de um método de análise multicritério e multiatores (MAMCA), envolveu planejadores e representantes de organizações não governamentais. Os resultados, obtidos por meio de workshops e consultas, mostraram que os atores envolvidos não possuíam leituras da realidade tão distintas entre si, embora o grupo de influenciadores tenha se mostrado mais crítico que o de planejadores. A infraestrutura cicloviária destacou-se como essencial para atrair ciclistas. A ausência de uma cultura da bicicleta também recebeu atenção. Contudo, mais significativo foi o fato de que o grupo de planejadores ignorou a importância da segurança de trânsito para o uso da bicicleta. Finalmente, a avaliação de cenários demonstrou que, para ambos os grupos, as medidas adotadas por São Paulo são pouco efetivas quando comparadas com outros casos.
\end{abstract}

\begin{abstract}
This study, which aims at identifying measures for cycling promotion in the city of São Paulo according to different stakeholders, involved planners and representatives of nongovernmental organizations by means of an adaptation of the Multi Actor Multi Criteria Analysis (MAMCA) approach. The results, obtained through workshops and questionnaires, showed that the perspectives of the two groups did not vary much, although the influencers were more critical than the planners. Cycling infrastructure was highlighted as key for attracting cyclists. The lack of a cycling culture was also an issue for both groups. However, the most significant point was the fact that planners ignored the role of traffic safety as a measure for cycling promotion. Finally, the evaluation of simplified scenarios showed that, for both groups, the measures adopted by the city of São Paulo were seen as ineffective when compared with other cases.
\end{abstract}

\section{INTRODUCÃO}

A bicicleta tem sido cada vez mais associada ao desenvolvimento de soluções sustentáveis para a mobilidade urbana. Contudo, sua introdução em ambientes pouco amigáveis aos ciclistas pode revelar-se um enorme desafio, como o exemplo da cidade de São Paulo (Benedini et al, 2019). A maior metrópole brasileira investiu ao longo da última década na criação de uma rede cicloviária buscando promover o uso da bicicleta. Nesse período, mais de 500 quilômetros de ciclovias foram construídos e sistemas de bicicletas compartilhadas iniciaram sua operação, recebendo o status de política pública (São Paulo, 2016). Tais ações promoveram um 
crescimento de $44 \%$ das viagens realizadas por bicicleta, ainda que a participação desse modo de transporte represente menos de 1\% do total de viagens (Metrô, 2019).

Apesar do aparente sucesso das medidas adotadas, mudanças políticas levaram à deterioração de parte da infraestrutura cicloviária, bem como à redução da área de cobertura dos serviços de compartilhamento. Este retrocesso levanta uma questão: estarão cidades de países em desenvolvimento preparadas para a adoção de soluções sustentáveis para os sistemas de transporte? A resposta a esta questão passa pela identificação de medidas que podem fomentar o uso da bicicleta como um modo regular de transporte. Esta resposta envolve, no entanto, a compreensão do problema a partir dos pontos de vista de atores distintos, dada sua natureza de política pública e, portanto, afeto a toda a sociedade.

Estudos que comparam as percepções de ciclistas e não ciclistas quanto às barreiras e motivações para o uso da bicicleta (Biernat et al., 2018; Iwińska et al., 2018; Félix et al., 2019) não são suficientes para capturar quais são os incentivos e impedimentos para a promoção do modo cicloviário. Dessa forma, esse estudo busca contribuir com as crescentes pesquisas para o entendimento dos fatores determinantes ao uso da bicicleta (seguindo o exemplo dos trabalhos de Irlam e Zuidgeest, 2018; Aldred et al., 2019).

Assim, o presente trabalho teve como objetivo identificar potenciais barreiras e fatores de incentivo ao uso da bicicleta a partir da comparação dos pontos de vista de diferentes stakeholders. Dada a natural dificuldade para a combinação de percepções de atores distintos, foi aplicado um método de Análise Multicritério e Multiatores (MAMCA), devidamente adaptado para atender às condições disponíveis para a realização desse estudo. A escolha de São Paulo foi motivada, entre outros fatores, pelo papel que a metrópole desempenha como referência para muitas outras cidades no país.

Esse documento é dividido em seis seções. Após a introdução, na seção dois encontra-se um resumo do que a literatura apresenta em termos de medidas para a promoção da bicicleta e também sobre o emprego da metodologia MAMCA. A abordagem metodológica considerando as adaptações promovidas é descrita na seção três, seguida pelos resultados (seção quatro) e pelas conclusões (seção cinco). Finalmente, a lista de referências citadas encerra o documento.

\section{REVISÃO DA LITERATURA}

Inúmeros trabalhos ao longo dos últimos anos buscaram identificar elementos associados à promoção do uso da bicicleta. A provisão de infraestrutura recebeu destaque por sua clara conexão com a atratividade de usuários (Basu e Vasudevan, 2013; Pucher et al., 2010; Sousa et al. 2014; Van Goeverden et al., 2015). Além disso, a segurança de ciclistas, diretamente associada à implantação de uma rede cicloviária também recebeu atenção (Marshall e Ferenchak, 2019; Pucher et al., 2010; Savan et at., 2017; Silva et al., 2014; Van Goeverden et al., 2015).

Características atribuídas às redes cicloviárias passaram a ser alvo de estudos que buscaram qualificar esses serviços, sempre associando sua atratividade a uma demanda crescente. Esse é o caso, por exemplo, das cycle superhighways na cidade de Londres. Segundo Li et al. (2018), essas rotas foram projetadas para atender à crescente demanda de ciclistas, especialmente daqueles provenientes dos sistemas de compartilhamento de bicicletas, os quais constituem uma parte importante das políticas de estímulo à uma mobilidade sustentável. Contudo, os autores afirmam que não há garantias de que tais infraestruturas ofereçam mais segurança a seus usuários, possivelmente por problemas relacionados a soluções incompletas associadas à falta de prioridade da bicicleta. Tal condição, por si só, pode limitar a atratividade de grupos 
diversificados de ciclistas. Esse exemplo mostra que a simples inserção de infraestrutura cicloviária parece possuir limitações quando se busca promover uma real transformação nos padrões de mobilidade urbana (Nkurunziza et al., 2012).

Harms et al. (2016) afirmam que há uma forte correlação entre o uso da bicicleta e o desenvolvimento de políticas flexíveis e de longo prazo desenvolvidas por múltiplos atores. Bons exemplos disso são Holanda e Dinamarca, os países onde proporcionalmente mais se usa a bicicleta no mundo (Pucher e Buehler, 2008). Para tal, empreenderam massivas políticas de transporte, uso do solo, desenvolvimento urbano, habitação, meio ambiente, tributárias e de estacionamentos, com especial atenção à criação de restrições para a circulação de automóveis. 0 estudo realizado por Van Goeverden et al. (2015), que analisa as políticas promovidas nesses mesmos países, identifica que ações diretas de governos nacionais foram essenciais para seus avanços, mesmo que políticas para a bicicleta sejam normalmente desenvolvidas em âmbito local. De fato, diversos autores vêm mostrando que intervenções sobre um único elemento não devem ser suficientes ou apenas levam a resultados marginais. Segundo Bertolini (2017), as políticas devem considerar intervenções coerentes sobre um amplo conjunto de componentes, incluindo não apenas a provisão de oferta e demanda, mas também sobre sistemas e seus contextos.

Também merecem destaque as barreiras impostas à adoção de pacotes de políticas para a promoção do modo cicloviário. Essa é uma questão que tem origem no não reconhecimento da bicicleta como um modo efetivo de transporte. Jones e Azevedo (2013) avaliam tal barreira como de grande relevância para a promoção da bicicleta no Brasil. Nesse sentido, Tennøy et al. (2015) criticam o quão distante o conhecimento técnico está dos processos de tomada de decisão ao se considerar a priorização dos modos não motorizados. Os autores avaliam que conhecimentos desatualizados e objetivos focados nos interesses de minorias reduzem as chances de se desenvolver projetos transformadores. Para Lyons e Davidson (2016), esse é o resultado de reduzida maturidade política, uma barreira para a construção de políticas de longa duração.

Dentre as alternativas para superar esses problemas, pressões criadas por grupos da sociedade podem ser efetivas, ainda que suas ações sejam limitadas (Jones e Azevedo, 2013; Assunção-Denis e Tomalty, 2018). É importante considerar que grupos políticos são influenciados por condicionantes culturais, o que significa que ações informativas e educativas podem mudar percepções, crenças e atitudes (Harms et al., 2016). Isso significa que acesso à educação, comunicação e informação são também necessários para promover o uso da bicicleta. Assim, campanhas representam uma forma direta de atrair usuários (Harms et al., 2016), ainda que tais práticas produzam resultados mais marginais do que a simples provisão de infraestrutura.

Os conceitos aqui apresentados reforçam a ideia de que o fomento ao uso da bicicleta é resultado de ações conjuntas que incluem implantação de infraestrutura, programas de incentivos, restrição ao uso do automóvel e também um planejamento coerente do uso do solo (Pucher et al., 2010). A importância de políticas adequadas é reforçada por Rietvel e Daniel (2004), que afirmam que decisões individuais tomadas por usuários são diretamente influenciadas por iniciativas promovidas por autoridades locais. Tais medidas resultam, no entanto, de decisões políticas originadas a partir de expectativas da própria sociedade (Bruhèze e Veraart, 1999). Dessa forma, compreender as diferentes visões sobre tais questões durante o processo de tomada de decisão pode ser uma forma de se garantir benefícios a todos os grupos, buscando o equilíbrio de interesses diversos (Freeman e McVea, 2001). 


\subsection{Análise multicritério e multiatores}

A compreensão das visões de atores distintos para o subsídio do processo de decisão não é tarefa fácil. Como esses nem sempre possuem perspectivas alinhadas, isto constitui um problema para o emprego de uma análise multicritério convencional. Métodos multicritério são normalmente utilizados durante o processo de tomada de decisão equilibrando pontos de vista, o que significa a avaliação de critérios a partir da atribuição de valores (pesos) médios, independentemente de possíveis discrepâncias verificadas entre os atores envolvidos. Quando o objetivo é o de se definir um ponto de vista único a partir de um grupo uniforme de stakeholders tal prática não representa um problema. Isso não ocorre, no entanto, quando é necessário levar em consideração visões distintas, especialmente sendo estas conflitantes.

Para contornar este problema, o método aqui empregado foi o MAMCA (do inglês Multi Actor Multi Criteria Analysis, ou análise multicritério e multiatores), desenvolvido por Macharis et al. (2012). A abordagem, que se propõe a minimizar conflitos no processo de tomada de decisão, já se mostrou útil em inúmeros problemas associados à temática dos transportes, tendo sido aplicado em casos das mais variadas naturezas, como os retratados por Dean e Hickman (2018), Roukouni et al. (2018), Van Lier et al. (2018) e Baudry e Vallée (2018).

0 envolvimento de inúmeros grupos não é tarefa simples, principalmente ao se considerar o consumo de tempo e demais custos envolvidos. Contudo, Cuppen et at. (2010) afirmam que o contato com perspectivas contraditórias dá aos atores a oportunidade não apenas de expressar seus próprios pontos de vista, mas também de levar em conta a opinião de outros grupos. A oposição de visões de stakeholders oferece perspectivas plurais, preferências e objetivos, dando ao processo de decisão a possibilidade de se tornar mais assertivo (Cuppen et at., 2010). Essa ideia é reforçada por Macharis e Baudry (2018), que consideram divergências entre tais grupos extremamente valiosas para tal.

O MAMCA não é, no entanto, apenas um método que incorpora divergências entre atores. Ele permite a definição e classificação de critérios segundo seu nível de importância para cada um dos grupos envolvidos, o que por sua vez leva à possibilidade de se comparar pontos de vista e valores. 0 método original consiste na aplicação de sete passos sucessivos. 0 primeiro deles corresponde à definição do problema e suas alternativas, que são apresentadas como diferentes cenários. No passo seguinte são identificados os grupos de stakeholders que são levados a avaliar o problema apresentado e considerar a importância relativa dos cenários frente a seus próprios critérios (passo 3). 0 quarto passo considera a definição de indicadores (quantitativos e/ou qualitativos) para avaliar a contribuição de cada uma das alternativas para atingir os critérios definidos. 0 quinto passo envolve o desenvolvimento das análises, que são seguidas pela extração dos resultados e análise de sensibilidade dos indicadores anteriormente propostos (passo 6). Finalmente, o sétimo e último passo inclui a implantação da alternativa escolhida, além das recomendações finais.

Embora esse estudo busque identificar as opiniões de atores distintos, ele não considera a definição de políticas a serem desenvolvidas e/ou implantadas. Isso significa que o potencial do MAMCA é explorado considerando uma análise pós-implantação. Assim, por meio da adaptação no método aqui proposta, os stakeholders podem avaliar soluções já adotadas, levando em conta critérios por eles identificados para a promoção do uso da bicicleta. 


\section{MÉTODO}

O método MAMCA - Multi Actor Multi Criteria Analysis (Macharis et al., 2012), que foi adaptado para uso neste estudo, possui forte emprego em avaliações de custo-benefício a partir de cenários com soluções propostas. Como não é este o caso, as modificações introduzidas nos procedimentos originais visam permitir a comparação de diferentes pontos de vista e a identificação de medidas e barreiras para o fomento do uso da bicicleta. Os passos adotados são apresentados a seguir.

\subsection{Definição do problema e identificação de alternativas}

O primeiro passo adotado consistiu na definição do problema e na identificação das alternativas (cenários). 0 problema a ser investigado consiste essencialmente em identificar medidas importantes para a promoção do uso da bicicleta em São Paulo a partir da consideração de distintos pontos de vista, ou seja, coincide com o objetivo deste estudo. Na versão original do método MAMCA, as alternativas devem variar de acordo com o problema, uma vez que se busca avaliar os custos e benefícios das soluções propostas para se encontrar a solução mais aceita por diferentes stakeholders. Contudo, um ponto chave para essa pesquisa consistiu na identificação de elementos que são essenciais para a promoção da bicicleta como um modo efetivo de transporte. Dessa forma, esse estudo apresentou uma abordagem exploratória de um problema que não possui soluções pré-definidas como alternativas para implantação.

Assim, a construção de cenários partiu da ideia de proporcionar uma avaliação de pacotes de políticas para a promoção do uso da bicicleta e não de casos já existentes. Contudo, a observação de casos reais teve utilidade para a identificação de medidas possíveis. Dessa forma, foram aqui incorporados três cenários fictícios (embora inspirados em conjuntos de ações efetivamente executadas em cidades reais). Um desses casos corresponde às medidas adotadas pela própria cidade de São Paulo. Dessa forma, buscaram-se informações que pudessem caracterizar as estratégias para políticas e infraestruturas realizadas nesta cidade. As seguintes características foram destaque: rede cicloviária desconectada com motivação inicial de lazer, baseada na adoção de ciclofaixas em detrimento de ciclovias, sem linearidade e diretividade, apresentando largura estreita e pavimentação inadequada; ausência de prioridade nas interseções e sinalização de trânsito sem clareza; segurança baseada na redução de velocidade sem a adoção de dispositivos de moderação de tráfego; reduzida conexão entre bicicletas e o transporte público; limitação à circulação do automóvel baseada no ciclismo de lazer representada por ciclofaixas nos domingos e feriados; sem programas para formação de uma cultura de respeito à bicicleta (Lemos et al., 2017; ITDP, 2015; Lemos e Wicher Neto, 2014).

Como a avaliação de um cenário único não faria sentido para esse estudo, outras alternativas foram incorporadas. Para tal, buscou-se um caso que pudesse representar o oposto de São Paulo. Assim, o exemplo das cidades holandesas foi considerado, dado seu reconhecimento internacional pelo sucesso ao incentivo à adoção da bicicleta. Dessa forma, um pacote de políticas com base nas medidas adotadas naquele país foi usado para moldar um cenário oposto ao da cidade brasileira. Dentre as medidas identificadas destacam-se: rede cicloviária extensa com garantia de prioridade e segurança, prioridade nas interseções, bypass em interseções semaforizadas; desenho viário com redução do raio para conversão de automóveis, adoção de inúmeros dispositivos de moderação de tráfego, barreiras físicas para limitação do acesso de automóveis e proibição de sua circulação especialmente nas áreas centrais; amplas áreas de estacionamento; forte cultura de respeito ao ciclista (Louw e Maat, 1999; Martens, 2007). 


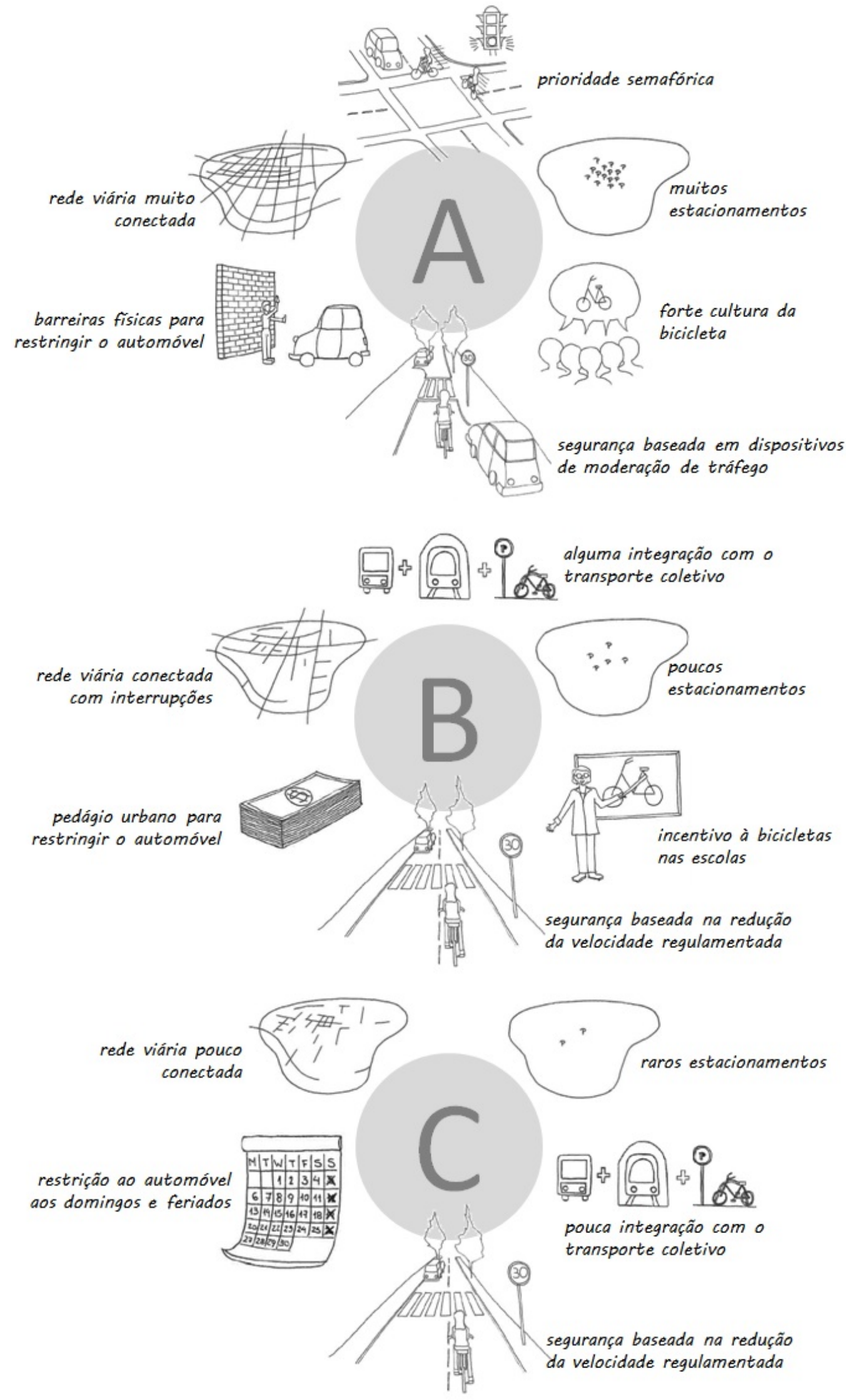

Figura 1. Cenário A, B e C (inspirados nos modelos da Holanda, Londres e São Paulo, respectivamente) Fonte: elaborado a partir dos estudos de Louw e Maat (1999), Martens (2007), London (2018), Lemos et al. (2017), ITDP (2015), Lemos e Wicher Neto (2014). 
Finalmente, para ampliar o conjunto de alternativas, decidiu-se pela inclusão de estratégia representando um modelo intermediário aos dois já definidos. A cidade de Londres, na Inglaterra, foi adotada para a constituição desse novo cenário, com destaque para as seguintes características: rede ampla (constituída principalmente por ciclofaixas), porém não completamente conectada, cycle superhighways conectando centro e periferia, falta de segurança em pontes, sem garantia de prioridade ao ciclista; segurança baseada na redução de velocidade, estacionamentos espalhados pela área central, porém em número insuficiente para atender à demanda; restrição à circulação do automóvel baseada no sistema de pedágio urbano; tentativa de integrar noções de comportamento no trânsito a partir de programa nas escolas (London, 2018). Tal escolha se deve ao fato de que, em termos de promoção da bicicleta, Londres está claramente à frente de São Paulo, porém atrás dos modelos verificados na Holanda.

Para a avaliação dos cenários propostos, os participantes desse estudo não tiveram acesso à correspondência dos cenários apresentados com os casos que lhes inspiraram. Eles apenas receberam um cartão ilustrando as principais estratégias e ações. Os casos foram divididos da seguinte forma: cenário A (inspirado no exemplo holandês), cenário B (inspirado na cidade de Londres) e cenário C (inspirado na cidade de São Paulo), conforme apresentado na Figura 1.

É importante destacar que esses cenários foram construídos a partir da identificação de ações baseadas em casos reais que representam diferentes níveis de ações em termos de promoção da bicicleta. Isso significa que as opções apresentadas foram estabelecidas para a identificação do nível de amadurecimento social e político frente à questão, desconsiderando, por exemplo, fatores de escala física e temporal. Dessa forma, não foi realizada uma avaliação da factibilidade de tais ações, mas sim de uma autocrítica a respeito de medidas que possam efetivamente levar ao uso efetivo da bicicleta.

\subsection{Análise de stakeholders}

O segundo passo envolveu a identificação dos grupos de stakeholders a serem considerados na cidade de São Paulo. Foram identificados potenciais participantes pertencentes a dois grupos: planejadores e influenciadores. 0 grupo dos planejadores foi constituído por tomadores de decisão em diferentes níveis de governo e representantes de empresas com conhecimento técnico na área. Por sua vez, o grupo de influenciadores foi formado por representantes de organizações não governamentais, que atuam para o estabelecimento de uma cultura da bicicleta em nível local, regional e nacional.

Os indivíduos, listados previamente, foram contatados por $e$-mail e/ou telefone e aqueles que demonstraram interesse e disponibilidade foram convidados a participar do estudo. Ao final, as atividades contaram com 11 participantes do grupo de planejadores representantes da Secretaria Municipal de Mobilidade e Transportes, Secretaria do Verde e Meio Ambiente, Companhia de Engenharia de Tráfego, Metrô, além de empresas de consultoria na área de transportes. 0 grupo dos influenciadores contou com nove participantes representantes de diferentes organizações da sociedade civil. Por razões de confidencialidade, nomes e organizações não foram aqui mencionados. Dois workshops foram realizados separadamente como forma de se evitar possíveis conflitos e constrangimentos. Para facilitar a subsequente análise dos dados, todas as atividades tiveram seus áudios gravados e transcritos, com a autorização dos participantes.

É importante destacar que os usuários (representantes da população que utilizam a bicicleta, mas que não atuam para sua promoção) não foram considerados nesse estudo. Essa escolha deu-se pelo fato de que esse consiste em um trabalho experimental para verificar a efetividade 
da metodologia MAMCA para identificação de elementos de fomento do uso da bicicleta, sem considerar uma análise a partir de soluções dadas, para a qual o método foi inicialmente desenvolvido. Nesse sentido, a opção foi dar preferência para planejadores e influenciadores que tendem a apresentar opiniões conflitantes, mas que indiretamente refletem a visão da sociedade sobre a bicicleta.

Segundo estudos de Bruhèze and Veraart (1999), as visões de técnicos, tomadores de decisão e de planejadores, bem como de organizações sociais, são diretamente correlacionadas à visão da sociedade a respeito da bicicleta. Para exemplificar essa teoria basta imaginar uma população que não possui uma cultura para o uso da bicicleta. Nesse caso, as ações governamentais acabam por refletir poucas ou nenhuma ação para esse fim. Assim, movimentos sociais identificam o problema e buscam influenciar tanto sociedade quanto poder público para gerar ações de promoção.

\subsection{Definição de critérios (medidas para a promoção da bicicleta)}

O passo seguinte consistiu na identificação dos critérios que resumissem a visão dos stakeholders sobre as medidas para promoção do uso da bicicleta em São Paulo. É importante levar em conta que, segundo a metodologia MAMCA, os critérios estabelecidos não são considerados efeitos ou impactos de ações, como normalmente observa-se nas análises multicritério. Nesse caso, eles representam os objetivos e ambições dos stakeholders envolvidos (Macharis et al., 2012).

Para a obtenção dos critérios foram realizados dois workshops separadamente com cada grupo de stakeholders. A partir de um roteiro simples, primeiro foram apresentados os objetivos e motivações desse estudo. Em seguida foi colocada uma pergunta chave aos presentes: "em sua opinião, que elementos podem contribuir para fomentar o uso da bicicleta?". Para respondê-la, os participantes foram divididos em grupos menores para debaterem opiniões com seus pares. Por fim, cada um dos grupos foi convidado a apresentar suas ideias, explicando suas motivações para a inserção das medidas apresentadas.

A análise dos discursos dos participantes buscou identificar os elementos por eles apontados como essenciais para o fomento do modo cicloviário. A nomenclatura adotada para os critérios considerou os termos usados originalmente pelos participantes. Esses foram em seguida agrupados em temas que buscaram conectar tópicos relacionados, conforme as justificativas dos próprios stakeholders para tal. Vale considerar que esse não é um procedimento obrigatório, mas que se mostrou um facilitador dada a grande quantidade de critérios apresentados.

Considerando que o objetivo desse estudo visa identificar tais elementos, os critérios estabelecidos não foram atribuídos buscando-se a construção de indicadores, um processo comum em análises multicritério. Assim, os elementos apontados pelos atores foram originalmente mantidos, representando suas ideias essenciais. Outra questão a ser considerada é que nem sempre os critérios apresentados pelos stakeholders são os mesmos. Tal característica é parte fundamental da metodologia MAMCA, a qual busca reforçar que nem sempre stakeholders estão alinhados e divergências são esperadas. Tal condição pode ser útil para a identificação dos pontos de concordância entre os atores envolvidos (Macharis e Baudry, 2018). 


\subsection{Definição de pesos para a avaliação de critérios}

Essa parte da metodologia MAMCA permite o reconhecimento das preferências específicas dos atores, ao mesmo tempo em que dá a eles a oportunidade de identificar o que é valorizado pelos demais (Macharis et al., 2018). Como resultado, foi possível verificar as prioridades de cada grupo, nesse caso relacionadas ao seu entendimento a respeito dos elementos para promoção do uso da bicicleta.

Para a obtenção dos pesos dos critérios foi utilizado o método Comparação Estruturada em Pares (da sigla em inglês SPC - Structured Pairwise Comparison). Descrito por Silva e Rodrigues da Silva (2009), Taleai et al. (2007) e Sharifi et al. (2006), esse é um procedimento de dois passos que se inicia com o ordenamento dos critérios segundo uma ordem de importância. A partir dos critérios ordenados, é realizada uma comparação entre pares de critérios como forma de se descrever as diferenças entre os mesmos. Assim, esse processo considera a atribuição de valores na comparação dos critérios, de forma que uma preferência fraca se torna uma unidade mais importante e uma preferência forte se torna duas unidades mais importante. Uma vantagem desse método está na interpretação direta da importância relativa dos critérios para participantes não familiarizados com processos mais complexos utilizados em análises multicritério, como as matrizes de avaliação par a par. Outra vantagem está na possibilidade de obtenção dos pesos por meio de questionários simples e on-line. Os pesos obtidos foram inseridos no MAMCA software, ferramenta utilizada para a análise dos dados levantados.

É importante destacar que, dada a natureza das informações coletadas (valores dos critérios de acordo com sua importância para os diferentes stakeholders), não foi realizada uma análise de sensibilidade de pesos. Tal procedimento possui grande utilidade em análises de fins concretos, como avaliação de projetos ou políticas a serem implementadas. Contudo, esse estudo não busca identificar as melhores soluções e sim pontos de vista, ainda que esses representem problemas para o desenvolvimento das ações esperadas (promoção do uso da bicicleta). De fato, no mundo real, conflitos e divergências são esperados entre grupos de stakeholders (Turcksin et al., 2011) e nem sempre as decisões são pautadas no melhor conhecimento técnico, mas sim em crenças e conceitos muitas vezes já superados (Tennøy et al., 2015). Esse, portanto, foi um dos objetivos que nortearam a escolha dessa ferramenta, dada sua capacidade de captar valores individualizados entre atores. Além disso, possíveis variações entre representantes do mesmo grupo de stakeholders foram dissipadas pela forma de tratamento de dados, que trabalhou com os valores médios dos pesos.

\subsection{Avaliação de cenários}

A metodologia MAMCA originalmente considera o emprego de indicadores como uma forma de se avaliar os cenários propostos, a partir da comparação com os critérios dados por stakeholders. Alternativamente, nesse estudo, os participantes foram levados a comparar diretamente os cenários com os temas (agrupamento de critérios) de forma a identificar quão efetivas são as soluções apontadas para a promoção da bicicleta. A ideia consistiu em dar aos atores a possibilidade de confrontar os elementos por eles apresentados (para o fomento ao uso da bicicleta) com as medidas já adotadas tanto no caso em estudo, quanto em outros modelos de iniciativas. 0 método utilizado para tal consistiu exatamente no mesmo anteriormente empregado para a obtenção dos pesos dos critérios, sendo inclusive parte integrante do mesmo formulário on-line enviado aos participantes. Os procedimentos adotados consideraram a avaliação que os grupos de planejadores e influenciadores fazem sobre os temas gerais, de 
acordo com sua eficácia na promoção do uso da bicicleta. A avaliação dos cenários a partir dos temas foi utilizada como forma de se reduzir o número de perguntas da pesquisa on-line, gerando um impacto positivo sobre o número final de questionários respondidos em sua totalidade. Assim como para a análise de pesos dos critérios, os dados obtidos para a avaliação de cenários também foram inseridos no MAMCA software.

\section{RESULTADOS}

A análise dos discursos de ambos os grupos apontou que, mesmo tendo sido questionados a respeito dos elementos positivos para o uso da bicicleta, as respostas foram apresentadas a partir do reforço de situações negativas no caso estudado. Isso sugere que, para os atores envolvidos, os elementos de estímulo ao uso da bicicleta são ao mesmo tempo as barreiras por eles enfrentadas. No total, 16 critérios foram apresentados (ver Tabela 1), sendo que todos foram citados pelos influenciadores e apenas 11 apareceram nos discursos dos planejadores. Destaca-se que os discursos apresentaram certa similaridade, de forma que os termos empregados para a definição dos critérios foram exatamente os mesmos entre grupos. Além disso, o agrupamento desses em temas se deu de maneira uniforme, ou seja, os dois grupos de stakeholders apresentaram a mesma configuração para os temas gerais: $\underline{\text { Cultura da bicicleta, }}$ Infraestrutura,

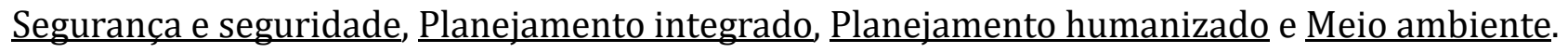

Tabela 1 - Critérios apresentados por grupos de stakeholders e seus pesos normalizados

\begin{tabular}{|c|c|c|c|}
\hline Temas & Critérios & Planejadores & Influenciadores \\
\hline \multirow[t]{4}{*}{ Cultura da bicicleta } & & 0.090 & 0.119 \\
\hline & Respeito aos ciclistas & 0.051 & 0.029 \\
\hline & Reconhecimento institucional do papel da bicicleta & - & 0.065 \\
\hline & Aprendizado sobre o uso da bicicleta na cidade & 0.039 & 0.025 \\
\hline \multirow[t]{4}{*}{ Infraestrutura } & & 0.434 & 0.345 \\
\hline & Rede cicloviária conectada & 0.242 & 0.183 \\
\hline & Sinalização de trânsito para ciclistas & 0.069 & 0.085 \\
\hline & Infraestrutura de apoio ao ciclista & 0.123 & 0.077 \\
\hline \multirow[t]{3}{*}{ Segurança e seguridade } & & 0.176 & 0.188 \\
\hline & Segurança de trânsito & - & 0.122 \\
\hline & Seguridade & 0.176 & 0.066 \\
\hline \multirow[t]{4}{*}{ Planejamento integrado } & & 0.110 & 0.183 \\
\hline & Integração entre o planejamento cicloviário e urbano & 0.042 & 0.082 \\
\hline & $\begin{array}{l}\text { Integração no planejamento de todos os modos de } \\
\text { transporte }\end{array}$ & 0.068 & 0.053 \\
\hline & Estratégias de desestímulo ao uso do automóvel & - & 0.048 \\
\hline \multirow[t]{3}{*}{ Planejamento humanizado } & & 0.110 & 0.091 \\
\hline & Planejamento orientado para pessoas & 0.079 & 0.056 \\
\hline & Respeito à opinião de ciclistas e não ciclistas & 0.031 & 0.035 \\
\hline \multirow[t]{4}{*}{ Meio ambiente } & & 0.080 & 0.074 \\
\hline & Qualidade do ar & 0.080 & 0.018 \\
\hline & Tipografia & - & 0.040 \\
\hline & Cobertura vegetal & - & 0.016 \\
\hline
\end{tabular}

A Cultura da bicicleta foi um tema que recebeu atenção a partir do entendimento de que este produz um impacto direto sobre a percepção da população a respeito do papel da bicicleta. Planejadores mencionaram o comportamento agressivo de motoristas como parte da cultura local, sendo essa uma justificativa importante para a adoção de ciclovias segregadas. Ao mesmo 
tempo, influenciadores apontaram que o governo falha em seu papel de comunicador e educador da população quanto à importância da disseminação da ideia da bicicleta. Portanto, para eles, é responsabilidade do poder público o mau entendimento que o cidadão possui a respeito do papel do ciclista para a mobilidade urbana. Tal visão reforça o conceito da formação de

uma cultura de uso da bicicleta apresentado por Bruhèze e Veraart (1999), que estabelece que a convergência de fatores físicos, políticos e culturais possui forte influência sobre a adoção e aceitação da bicicleta como modo efetivo de transporte.

0 tema Infraestrutura foi frequentemente associado à precariedade da rede cicloviária, bem como à falta de atendimento às necessidades básicas dos ciclistas, como locais seguros para estacionamento, além de sinalização de trânsito específica. Para ambos os grupos, a ausência de infraestrutura adequada, como uma rede conectada, representa uma barreira significativa à adoção da bicicleta.

Segurança e seguridade receberam duas abordagens distintas, a primeira está associada ao conceito de segurança de tráfego e a segunda à violência urbana. Ambos são essenciais para a atratividade de ciclistas e caso a percepção da população seja afetada por qualquer um deles, as chances de uso da bicicleta são demasiado restritas. Apesar disso, o grupo de planejadores não citou Segurança como um elemento para a promoção da bicicleta. Surpreendentemente ele foi esquecido, ou simplesmente não mencionado, por representantes do poder público e técnicos da área. Além de surpreendente, isso representa um alerta para a cidade de São Paulo, especialmente após o aumento de $64 \%$ no número de ciclistas mortos em acidentes de trânsito no último ano, segundo dados do Infosiga (São Paulo, 2020). Por outro lado, os influenciadores apontaram que medidas de moderação de tráfego são fundamentais para amenizar os problemas de segurança no trânsito. Para ambos os grupos, a maioria da população percebe Seguridade como uma barreira natural ao uso da bicicleta sendo que, ao entendimento dos participantes, esse parece ser um problema insolúvel.

O Planejamento integrado, tema que considera a associação entre estratégias cicloviárias e demais modos de transporte foi apresentado a partir de fortes críticas à forma como a cidade organiza suas ações no território. Os participantes reforçaram que a realização de ações fragmentadas, em frentes distintas (transporte público, gerenciamento de tráfego, ações para pedestres, planejamento cicloviário e desenho urbano), promovem soluções inadequadas para a cidade, dado que o espaço urbano é um só e todos os elementos interagem entre si. Assim os dois grupos de stakeholders avaliaram que há uma clara dissociação entre o planejamento de transportes e o ambiente urbano, sendo essa uma grande barreira para o uso efetivo da bicicleta como modo de transporte. Até mesmo a Integração entre todos os modos de transporte foi apontada como uma barreira.

Também recebeu atenção o tema denominado Planejamento humanizado há algum tempo discutido na temática do planejamento urbano. Os participantes mostraram clara referência ao conceito de planejamento "orientado para as pessoas", que foi definido por Chen et al. (2019) como uma visão humanizada sobre os processos de planejamento. Na prática, isso significa que o planejamento humanizado está associado à melhoria da qualidade de vida no ambiente construído, a partir de uma visão centrada nas necessidades dos indivíduos. Em São Paulo, os stakeholders reforçaram a urgência na adoção desse enfoque, evitando soluções massivas que ignoram os problemas enfrentados pela população. 
O último tema presente nas falas dos grupos participantes consistiu em Meio ambiente. Influenciadores mencionaram que a desconsideração aos condicionantes ambientais na execução de estratégias para o uso da bicicleta representa uma barreira para a atratividade de ciclistas. Escolhas inadequadas para o traçado de rotas cicloviárias em vias com Topografia pouco amigável e a falta de Cobertura vegetal são exemplos disso. Além disso, a Poluição do ar foi lembrada por ambos os grupos como uma barreira para a atratividade de ciclistas. Tais elementos causam dúvidas quanto à sua inclusão para a promoção da bicicleta. Questões topográficas e cobertura vegetal podem ser associadas à natureza da infraestrutura. Entretanto, a ênfase dos participantes em atribuir a esses elementos características dissociadas da infraestrutura levou à decisão de mantê-los em agrupamento próprio.

\subsection{Avaliação de critérios}

Os critérios foram avaliados individualmente pelos stakeholders seguindo sua percepção quanto à eficiência para atingir o objetivo desejado. Esse processo se deu a partir do envio de uma pesquisa por e-mail para cada um dos participantes. Os pesos normalizados para os critérios, obtidos pelo método SPC, representaram os valores médios atribuídos entre planejadores e influenciadores, sendo esses também identificados na Tabela 1. Os resultados podem ser usados para a identificação não apenas da relevância individualizada de cada critério, mas também dos respectivos temas (ver Figura 2). Considerando-se os pesos acumulados por temas gerais, torna-se evidente o valor atribuído à Infraestrutura como medida essencial para fomentar a bicicleta como modo efetivo de transporte.

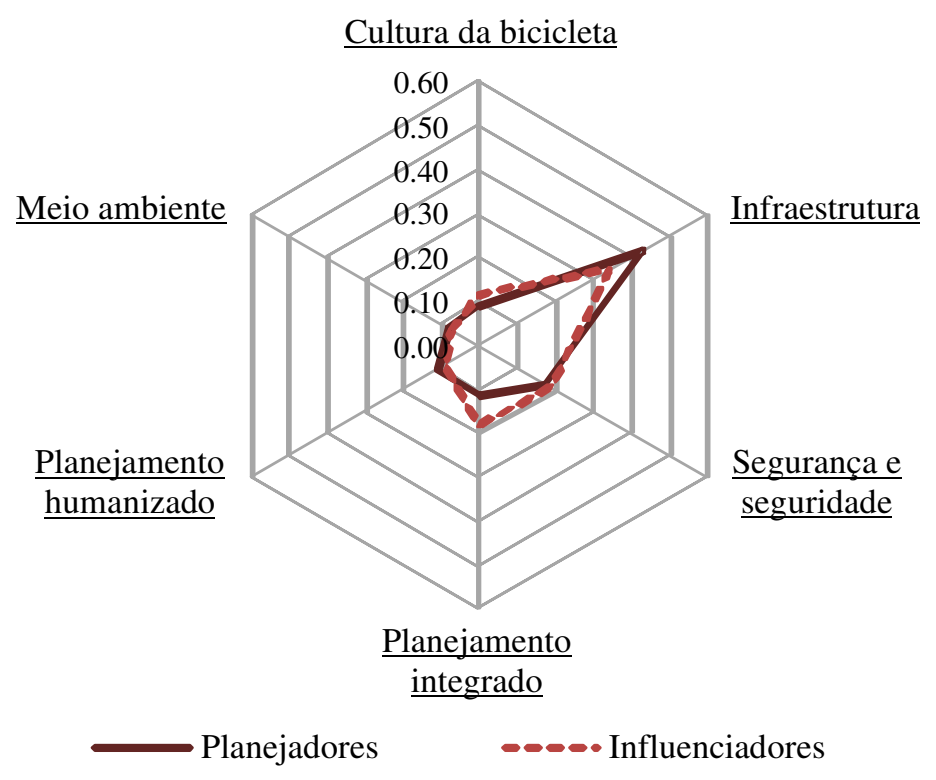

Figura 2. Pesos normalizados por temas gerais para cada grupo de stakeholders

Um dos processos de avaliação sugeridos pela metodologia MAMCA considera a comparação dos pesos dos critérios identificados simultaneamente pelos diferentes grupos de atores, considerando que esses possuem valor para todos os indivíduos e, portanto, podem ser diretamente comparados. A Figura 3 apresenta esse resultado.

0 critério Rede cicloviária conectada, pertencente ao tema Infraestrutura, foi muito bem 
avaliado pelos dois grupos. Essa condição reforça a importância atribuída ao referido tema para a atração de ciclistas. Além disso, é notável o destaque atribuído à Seguridade por planejadores, embora o mesmo grupo tenha ignorado a Segurança (razão pela qual o critério não é representado na Figura 3). Destaca-se o fato de que ao mesmo tempo em que planejadores atribuíram grande peso à questão da segurança pública para a escolha do modo cicloviário, esse é um problema percebido pelo grupo como uma barreira natural e, portanto, insolúvel. Tal condição pode ser interpretada como a aceitação de que o grupo não percebe sua responsabilidade quanto à questão e que, dessa forma, nada tem a fazer para solucioná-la.

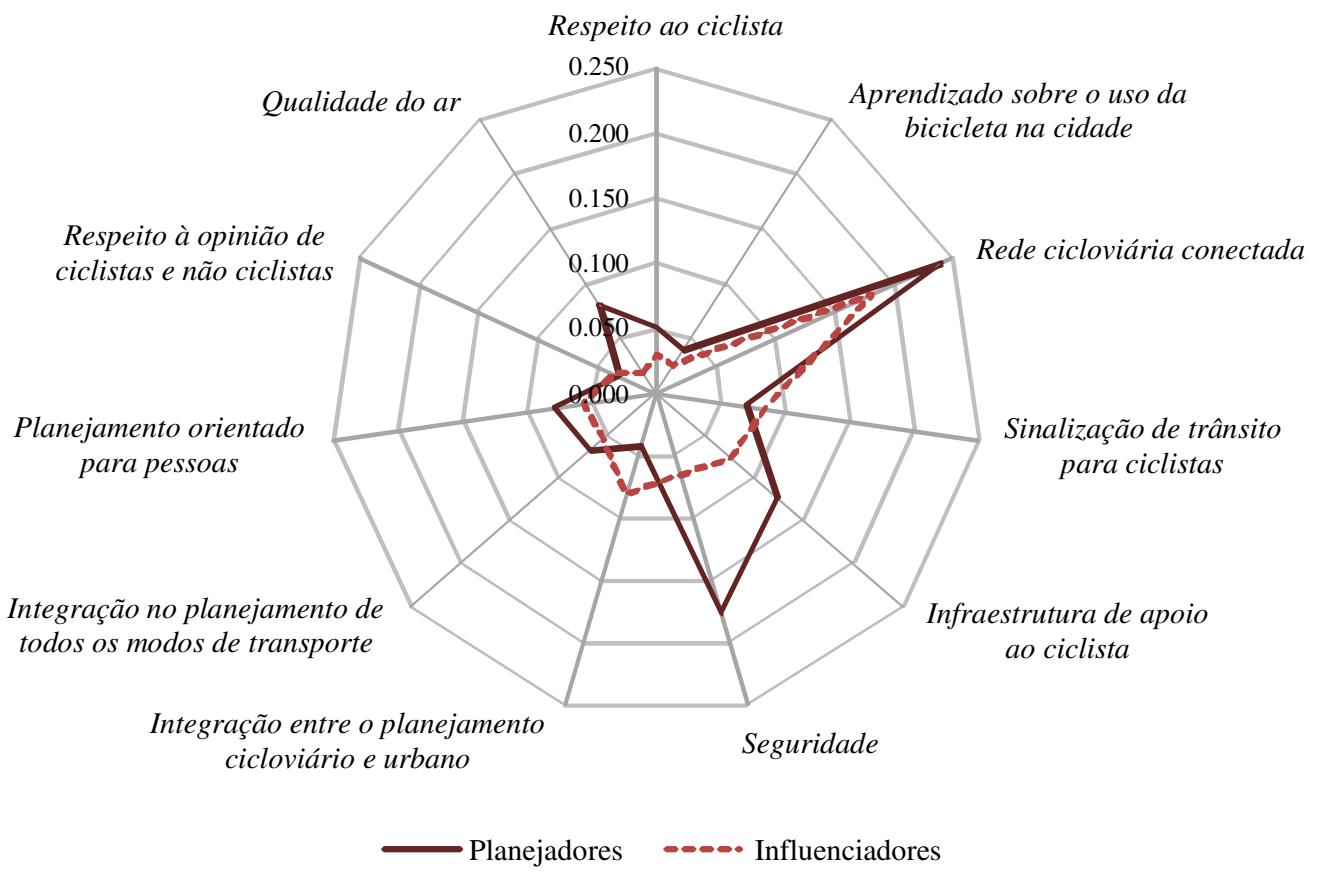

Figura 3. Pesos normalizados dos critérios atribuídos por planejadores e influenciadores

\subsection{Avaliação de cenários}

Os dois grupos de stakeholders foram levados a avaliar os conjuntos de soluções para o fomento do uso da bicicleta, representados por três cenários. Esse procedimento seguiu a metodologia MAMCA, que confronta a efetividade de cada cenário em relação aos temas. 0 método de avaliação foi o mesmo usado para obter os pesos dos critérios, como parte do questionário online enviado aos participantes. Dessa forma, planejadores e influenciadores avaliaram os cenários frente aos temas e não aos critérios individualizados.

Para cada um dos temas, os stakeholders classificaram os cenários A, B e C de acordo com sua eficácia quanto à promoção do uso da bicicleta. Os resultados obtidos nesse processo podem ser observados na Figura 4. O cenário A (inspirado no caso holandês) recebeu a melhor avaliação para todos os temas, tanto por planejadores quanto influenciadores. Além disso, houve total concordância quanto ao fraco desempenho das medidas adotadas para o fomento do uso da bicicleta exibidas no cenário C, inspirado na cidade de São Paulo. Tal resultado indica que sequer o grupo de stakeholders responsável pela concretização das medidas para o uso da bicicleta na própria cidade concordou que suas ações sejam de fato efetivas, quando comparadas com medidas empreendidas em outros locais. 
0 resultado dessa análise pode parecer óbvio. Certamente planejadores e influenciadores identificam no cenário baseado no modelo holandês maior efetividade para a promoção da bicicleta. No entanto, dada a natureza desse estudo, que busca lançar uma luz sobre diferentes pontos de vista e não sobre as melhores soluções, tal conclusão aponta para uma questão importante: se há uma concordância sobre a eficiência de determinado modelo, por que esse não é adotado?

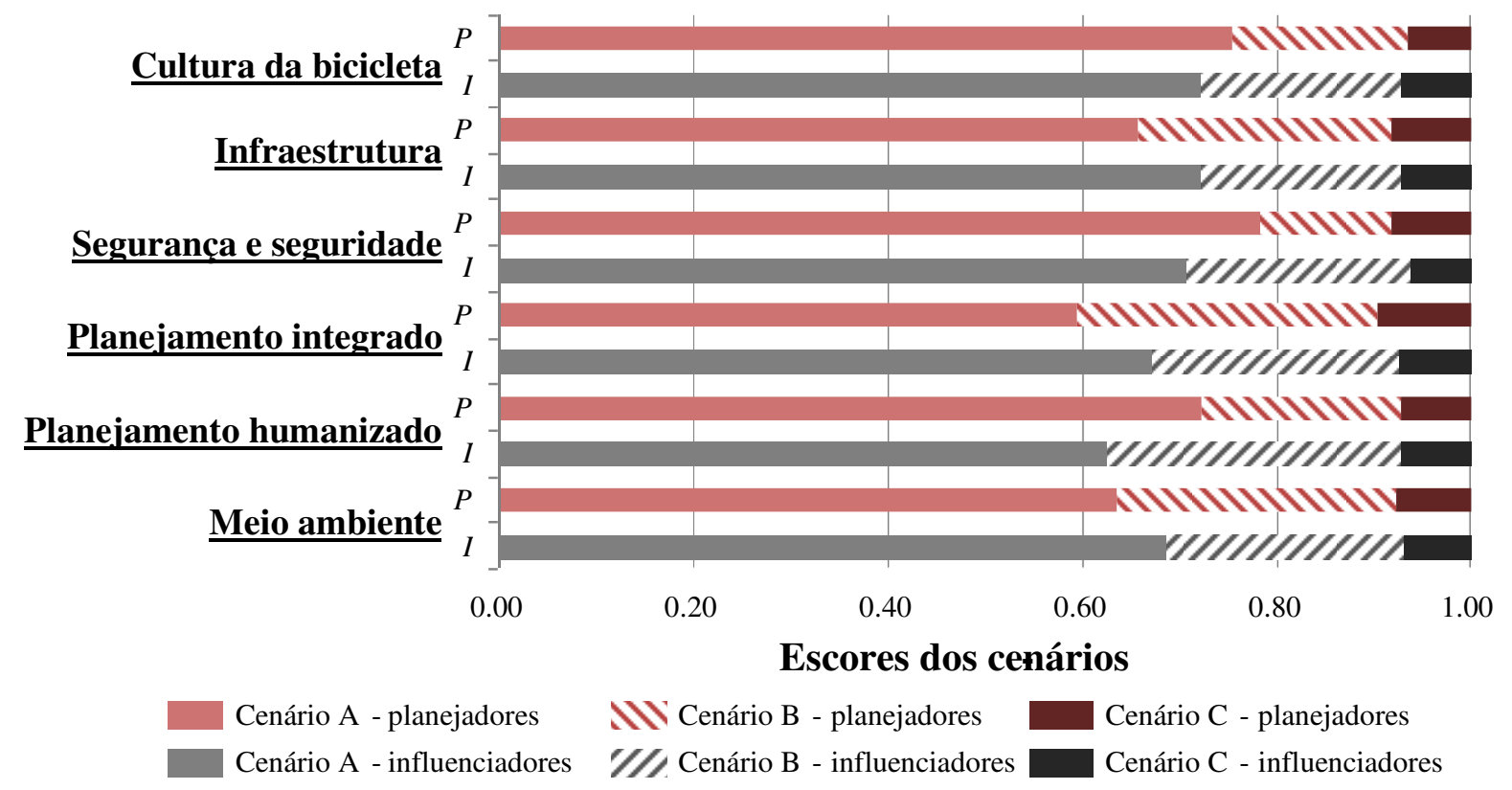

Figura 4. Avaliação de cenários por planejadores e influenciadores

Essa questão leva ao questionamento sobre o distanciamento entre o conhecimento técnico e a tomada de decisão. A literatura aponta que decisões políticas se sobrepõem ao conhecimento técnico e assim acabam ferindo os interesses da sociedade, como apontado por Withanaarachchi e Setunge (2014). Dessa forma, o reconhecimento dos atores sobre as medidas frágeis implantadas na cidade de São Paulo mostra que a adoção de políticas transformadoras exige mudanças mais profundas do que a simples apropriação de conhecimento técnico.

\section{CONCLUSÕES}

A aplicação de uma metodologia baseada na perspectiva de múltiplos atores mostrou-se adequada para a identificação dos elementos apontados por esses como essenciais para o fomento do uso da bicicleta, considerando-se simultaneamente diferentes pontos de vista. Os discursos apresentados pelos participantes dos workshops realizados na cidade de São Paulo foram pautados em uma visão negativa da questão. As respostas dadas por planejadores e influenciadores, quando questionados a respeito dos elementos por eles considerados essenciais para a promoção da bicicleta, foram apresentadas a partir de uma visão crítica dos problemas associados à condição da bicicleta como um modo efetivo de transporte.

Os dados coletados com os grupos de stakeholders mostraram que as visões desses a respeito dos elementos para o fomento do uso da bicicleta não são tão diferentes. Os elementos identificados por ambos foram bastante similares, embora influenciadores tenham explorado tópicos não abordados por planejadores. Para ambos os grupos de atores, a infraestrutura 
recebeu destaque por seu papel essencial para a atratividade da bicicleta. Essa é uma condição já explorada por trabalhos anteriores, que reforça a importância de uma rede cicloviária para garantir a prioridade e segurança dos ciclistas (Pucher et al., 2010; Basu e Vasudevan, 2013; Sousa et al., 2014; Van Goeverden et al., 2015).

Os discursos foram críticos quanto aos processos de planejamento e implantação das ações locais, o que para os participantes indica outro grave problema: a ausência de uma cultura da bicicleta. Segundo influenciadores, isso reflete a incapacidade do governo de comunicar e educar a população sobre o papel da bicicleta. No entanto, o ponto mais significativo a ser destacado consiste no fato de que o grupo de planejadores ignorou segurança de trânsito como um critério de atratividade.

Por sua vez, a visão negativa manifestada pelos atores locais foi refletida na forma como tais grupos avaliaram o conjunto de estratégias e ações representando o exemplo de São Paulo (cenário C). $\mathrm{O}$ confronto dos grandes temas com os cenários apresentados demonstra que tanto planejadores quando influenciadores identificaram como ineficazes as medidas adotadas na cidade para o fomento do uso da bicicleta. Merece destaque o fato de que tais atores, embora diretamente associados às iniciativas adotadas, não reconhecem os próprios esforços como suficientes para ampliar o uso da bicicleta na cidade. Nesse sentido, identifica-se a importância de descobrir quais são os passos necessários para vencer tais resistências e, assim, construir uma efetiva cultura de valorização do modo cicloviário.

Vale lembrar que este estudo possui limitações, pois foi desenvolvido a partir da visão de apenas dois grupos de stakeholders. Recomenda-se sua aplicação a outros grupos da sociedade, tais como representantes de empresas operadoras de serviços de bicicletas compartilhadas, bem como dos próprios usuários desses sistemas/serviços. Além disso, a extensão da análise para outros casos, sobretudo de exemplos bem sucedidos para a promoção da bicicleta, pode levar a uma interessante comparação entre a opinião de stakeholders de realidades distintas.

\section{AGRADECIMENTOS}

Essa pesquisa recebeu o apoio da CAPES (Coordenação de Aperfeiçoamento de Pessoal de Nível Superior - Código de Financiamento 001), da FAPESP (Fundacão de Amparo à Pesquisa do Estado de São Paulo, Processo 2015/50129-5 - Projeto DePICT), do CNPq (Conselho Nacional de Desenvolvimento Científico e Tecnológico, Processos 308436/2015-6 e 140484/2020-5) e do MAMCA Software (VUB-MOBI group).

\section{REFERÊNCIAS}

Aldred, R.; T. Watson; R. Lovelace e J. Woodcock (2019) Barriers to investing in cycling: Stakeholder views from England. Transportation Research Part A, v. 128, p. 149 1-159. DOI: 10.1016/j.tra.2017.11.003.

Assunçao-Denis, M. e R. Tomalty (2018) Increasing cycling for transportation in Canadian communities: Understanding what works. Transportation Research Part A, v. 123, p. 288-304. DOI: 10.1016/j.tra.2018.11.010.

Basu, S. e V. Vasudevan (2013) Effect of bicycle friendly roadway infrastructure on bicycling activities in urban India. Procedia - Social and Behavioral Sciences, v. 104, p. 1139-1148. DOI: 10.1016/j.sbspro.2013.11.210.

Baudry, G. e T. Vallée (2018) Assessing the stakeholder support for different biofuel options in France by 2030 using the range-based Multi actor Multi Criteria Analysis framework. In C. Macharis \& G. Baudry (Eds.), Decision-making for sustainable transport and mobility, p. 2-27. Cheltenham, UK: Edward Elgar Publishing Limited, Inc.

Benedini, D. J.; P. S. Lavieri e O. Strambi (2019) Understanding the use of private and shared bicycles in large emerging cities: The case of São Paulo, Brazil. Case Studies on Transport Policy. v. 8, n. 2, p. 564-575. DOI: 10.1016/j.cstp.2019.11.009.

Bertolini, L. (2017). Planning the mobile metropolis. Transport for people, places and the planet. Palgrave, Macmillan Publishers Limited, London.

Biernat, E.; S. Buchholtz e P. Bartkiewicz (2018) Motivations and barriers to bicycle commuting: Lessons from Poland. Transportation Research Part F, v. 55, p. 492-502. DOI: 10.1016/j.trf.2018.03.024.

Bruhèze, A. A. A. e F. C. A. Veraart (1999) Fietsverkeer in praktijk en beleid in de twintigste eeuw: overeenkomsten en verschillen in fietsgebruik in Amsterdam, Eindhoven, Enschede, Zuidoost-Limburg, Antwerpen, Manchester, Kopenhagen, Hannover en Basel. Ministerie van Verkeer en Waterstaat, Rijkswaterstaat, Hoofdkantoor van de Waterstaat, Den Haag. 
Chen, M.; Y. Gong; D. Lu e C. Ye (2019) Build a people-oriented urbanization: China's new-type urbanization dream and Anhui model. Land Use Policy, v. 80, p. 1-9. DOI: 10.1016/j.landusepol.2018.09.031.

Cuppen, E.; S. Breukers; M. Hisschemöller e E. Bergsma (2010) Q methodology to select participants for a stakeholder dialogue on energy options from biomass in the Netherlands. Ecological Economics, v. 69, n. 3, p. 579-591. DOI: 10.1016/j.ecolecon.2009.09.005.

Dean, M. e R. Hickman (2018) Comparing Cost-Benefit Analysis and Multi Actor Multi Criteria Analysis: the case of Blackpool and the South Fylde Line. In Macharis, C. e G. Baudry (Eds.), Decision-making for sustainable transport and mobility, p. 227. Cheltenham, UK: Edward Elgar Publishing Limited, Inc.

Félix, R.; F. Moura e K. J. Clifton (2019) Maturing urban cycling: Comparing barriers and motivators to bicycle of cyclists and non-cyclists in Lisbon, Portugal. Journal of Transport \& Health, v. 15, 100628. DOI: 10.1016/j.jth.2019.100628.

Freeman, R. E. e J. A. McVea (2001) Stakeholder approach to strategic management. Darden Business School Working Paper, n. 01-02. DOI: $10.2139 /$ ssrn.263511.

Harms, L.; L. Bertolini e M. te Brömmelstroet (2016) Performance of municipal cycling policies in medium-sized cities in the Netherlands since 2000. Transport Reviews, v. 36, n. 1, 134-162. DOI: 10.1080/01441647.2015.1059380.

Irlam, J. H. e M. Zuidgeest (2018) Barriers to cycling mobility in a low-income community in Cape Town: A Best-Worst Scaling approach. Case Studies on Transport Policy, v. 6, n. 4, p. 815-823. DOI: 10.1016/j.cstp.2018.10.003.

Iwińska, K.; M. Blicharska; L. Pierotti; M. Tainio e A. Nazelle (2018) Cycling in Warsaw, Poland - Perceived enablers and barriers according to cyclists and non-cyclists. Transportation Research Part A - Policy Pract, v. 113, p. 291-301. DOI: 10.1016/j.tra.2018.04.014.

ITDP (2015) Políticas de mobilidade por bicicleta e rede cicloviária da cidade de São Paulo: análise e recomendações. Instituto de Políticas de Transporte \& Desenvolvimento. Disponível em: <http://itdpbrasil.org.br/wpcontent/uploads/2016/03/Relatorio-CIclovias-SP.pdf>. (Acesso em: 06/06/2019).

Jones, T. e L. N. Azevedo (2013) Economic, social and cultural transformation and the role of the bicycle in Brazil. Journal of Transport Geography, v. 30, p. 208-219. DOI: 10.1016/j.jtrangeo.2013.02.005.

Lemos, L.; M. K. Harkot; P. F. Santoro e I. B. Ramos (2017) Mulheres, por que não pedalam? Por que há menos mulheres do que homens usando bicicleta em São Paulo, Brasil? Revista Transporte y Territorio, v. 16, p. 68-98. ISSN 1852-7175.

Lemos, L.e H. Wicher Neto (2014) Cycling infrastructure in São Paulo: Impacts of a leisure-oriented model. In: Spinoffs of Mobility: Technology, Risks \& Innovation - 12th Annual Conference of the International Association for the History of Transport, Traffic and Mobility $\left(T^{2} M\right)$, Filadélfia. Spinoffs of Mobility: Technology, Risks \& Innovation.

Li, H.; H. Ding; G. Ren e C. Xu (2018) Effects of the London cycle superhighways on the usage of the London cycle hire. Transportation Research, v. 111, p. 304-315. DOI: 10.1016/j.tra.2018.03.020.

London (2018) Mayor's Transport Strategy. Greater London Authority, London, UK.

Louw, E. e K. Maat (1999) Enschede: measures in a package. Built Environment, Travel Reduction: Policy into Practice, v. 25, p. 118-128.

Lyons, G. e C. Davidson (2016) Guidance for transport planning and policymaking in the face of an uncertain future. Transportation Research, v. 88, p. 104-116. DOI: 10.1016/j.tra.2016.03.012.

Macharis, C.; L. Turcksin e K. Lebeau (2012) Multi actor multi criteria analysis (MAMCA) as a tool to support sustainable decisions: state of use. Decision Support Systems, v. 54, n. 1, p. 610-620. DOI: 10.1016/j.dss.2012.08.008.

Macharis, C. e G. Baudry (2018) The Multi Actor Multi Criteria Analysis framework. In Macharis, C. e G. Baudry (Eds.), Decision-making for sustainable transport and mobility, p. 2-27. Cheltenham, UK: Edward Elgar Publishing Limited, Inc.

Macharis, C.; K. de Brucker e K. van Raemdonck (2018) When to use Multi Actor Multi Criteria Analysis or other evaluation methods? In Macharis, C. e G. Baudry (Eds.), Decision-making for sustainable transport and mobility, p. 28-47. Cheltenham, UK: Edward Elgar Publishing Limited, Inc.

Marshall, W. E. e N. F. Ferenchak (2019) Why cities with high bicycling rates are safer for all road users. Journal of Transport \& Health, v. 13, 100539. DOI: 10.1016/j.jth.2019.03.004.

Martens, K. (2007) Promoting bike-and-ride: The Dutch experience. Transportation Research Part A, v. 41, n. 4, p. $326-338$. DOI:10.1016/j.tra.2006.09.010.

Metrô (2019) Pesquisa Origem Destino 2017. Relatório Técnico, Companhia do Metropolitano de São Paulo, São Paulo.

Nkurunziza, A.; M. Zuidgeest; M. Brussel e M. van Maarseveen (2012) Examining the potential for modal change: Motivators and barriers for bicycle commuting in Dar-es-Salaam. Transport Policy, v. 24, p. 249-259. DOI: 10.1016/j.tranpol.2012.09.002.

Pucher, J. e R. Buehler (2008) Making cycling irresistible: Lessons from the Netherlands, Denmark and Germany. Transport Reviews, v. 28, n. 4, p. 495-528. DOI: 10.1080/01441640701806612.

Pucher, J.; J. Dill e S. Handy (2010) Infrastructure, programs, and policies to increase bicycling: An international review. Preventive Medicine, v. 50, p. S106-S125. DOI: 10.1016/j.ypmed.2009.07.028.

Rietveld, P. e V. Daniel (2004) Determinants of bicycle use: do municipal policies matter? Transportation Research, v. 38, i. 7, p. 531-550. DOI: 10.1016/j.tra.2004.05.003.

Roukouni, A.; C. Macharis e S. Basbas (2018) Evaluation of value capture financing schemes for urban transportation infrastructure with the aid of Multi Actor Multi Criteria Analysis focusing on a Greek city. In Macharis, C. e G. Baudry (Eds.), Decision-making for sustainable transport and mobility, p. 2-27. Cheltenham, UK: Edward Elgar Publishing Limited, Inc. 
São Paulo (2020) Infosiga, Base de dados. Governo do Estado de São Paulo, São Paulo. Disponível em: <http://www.respeitoavida.sp.gov.br/>. (Acesso em: 02/02/2020).

São Paulo (2016) Decreto municipal no ${ }^{\circ}$ 56.834, de 24 de fevereiro de 2016. Institui o Plano Municipal de Mobilidade Urbana de São Paulo - PlanMob/SP 2015, Município de São Paulo.

Savan, B.; E. Cohlmeyer e T. Ledsham (2017) Integrated strategies to accelerate the adoption of cycling for transportation. Transportation Research A, v. 46, p. 236-249. DOI: 10.1016/j.trf.2017.03.002.

Sharifi, M. A.; L. Boerboom; K. B. Shamsudin e L. Veeramuthu (2006) Spatial multiple criteria decision analysis in integrated planning for public transport and land use development study in Klang Valley, Malaysia. ISPRS Technical Commission II Symposium, Vienna, p. 12-14.

Silva. A.; I. Pinto; D. Ribeiro e J. Delgado (2014) Multicriteria analysis for evaluation of bike lane routes integrated to public transportation. Procedia - Social and Behavioral Sciences, v. 162, p. 388-397. DOI: 10.1016/j.sbspro.2014.12.220.

Silva, L. M. e A. N. Rodrigues da Silva (2009) Planejamento estratégico de uma experiência pedagógica inovadora. Pesquisa e Tecnologia Minerva, v. 6, n. 1, p. 99-106.

Sousa, A. A.; S. Sanches e M. A. G. Ferreira (2014) Perception of barriers for the use of bicycles. Procedia - Social and Behavioral Sciences, v. 160, p. 304-313. DOI: 10.1016/j.sbspro.2014.12.142.

Taleai, M.; A. Sharifi; R. Sliuzas e M. Mesgari (2007) Evaluating the compatibility of multi-functional and intensive urban land uses. International Journal of Applied Earth Observation and Geoinformation, v. 9, n. 4, p. 375-391. DOI: 10.1016/j.jag.2006.12.002.

Tennøy, A.; L. Hansson; E. Lissandrello e P. Næss (2015) How planners' use and non-use of expert knowledge affect the goal achievement potential of plans: experiences from strategic land-use and transport planning processes in three Scandinavian cities. Progress in Planning, v. 109, p. 1-32. DOI: 10.1016/j.progress.2015.05.002.

Turcksin, L.; C. Macharis; K. Lebeau; F. Boureima; J. van Mierlo; S. Bram; J. de Ruyck; L. Mertens; J. Jossart; L. Gorissen e L. Pelkmans (2011) A multi-actor multi-criteria framework to assess the stakeholder support for different biofuel options: The case of Belgium. Energy Policy, v. 39, n. 1, p. 200-214. DOI: 10.1016/j.enpol.2010.09.033.

Van Goeverden, K.; T. S. Nielsen; H. Harder e R. van Nes (2015) Interventions in bicycle infrastructure, lessons from Dutch and Danish cases. Transportation Research Procedia, v. 10, p. 403-412. DOI: 10.1016/j.trpro.2015.09.090.

Van Lier, T.; D. Meers; H. B. Rai e C. Macharis (2018) Evaluating innovative solutions for sustainable city logistics: an enhanced understanding of stakeholders perceptions. In Macharis, C. e G. Baudry (Eds.), Decision-making for sustainable transport and mobility, p. 2-27. Cheltenham, UK: Edward Elgar Publishing Limited, Inc.

Withanaarachchi, J. e S. Setunge (2014) Decision making on transport network planning and the impact on community, economy and the environment. Procedia Economics and Finance, v. 18, p. 882-891. DOI: 10.1016/S2212-5671(14)010144. 